\title{
Regulation of Niche Molecules for the Maintenance of Cardiac Stem Cells
}

\author{
Nobuyuki Takakura, MD, PhD
}

$\mathbf{T}$ issue/organ specific stem cells play central roles in the formation, development and maintenance of most organs and are thought to be crucial cell populations for tissue regeneration therapies. The first definitively identified stem cell populations were the hematopoietic stem cells (HSCs). Since then, many stem cell populations have been isolated and gradually tissue/organ-specific stem cells have been identified in diverse organs such as brain, skin and colon. A single stem cell generates large numbers of terminally-differentiated tissue/organ-specific cells via its differentiation into progenitor cells; however, it must retain the ability to divide asymmetrically, whereby 1 daughter cell retains stemness. The latter event is designated self-renewal and, by means of this property of stem cells, turnover of tissue-specific cells is maintained.

\begin{tabular}{l} 
Article p222 \\
\hline Bone marrow (BM) transplantation was the first application \\
utilizing stem cells for tissue regeneration therapy, exploiting \\
the function of HSCs for differentiation into all cells of the \\
hematopoietic lineage. BM reconstitution is maintained long \\
term by the self-renewal activity of HSCs. Transfusions of red \\
blood cells or platelets remain important for transient treatment \\
of hematopoietic deficiencies, but stem cell transplantation is \\
required for whole tissue/organ regeneration. At the present \\
time, methods to generate tissue/organ-specific terminally- \\
differentiated cells from immature cell types, such as ES and \\
iPS cells, have been established; we need to keep in mind that
\end{tabular}

\section{Article p 222}

\begin{abstract}
Bone marrow (BM) transplantation was the first application utilizing stem cells for tissue regeneration therapy, exploiting the function of HSCs for differentiation into all cells of the hematopoietic lineage. BM reconstitution is maintained long term by the self-renewal activity of HSCs. Transfusions of red blood cells or platelets remain important for transient treatment iPS cells, have been established; we need to keep in mind that
\end{abstract}

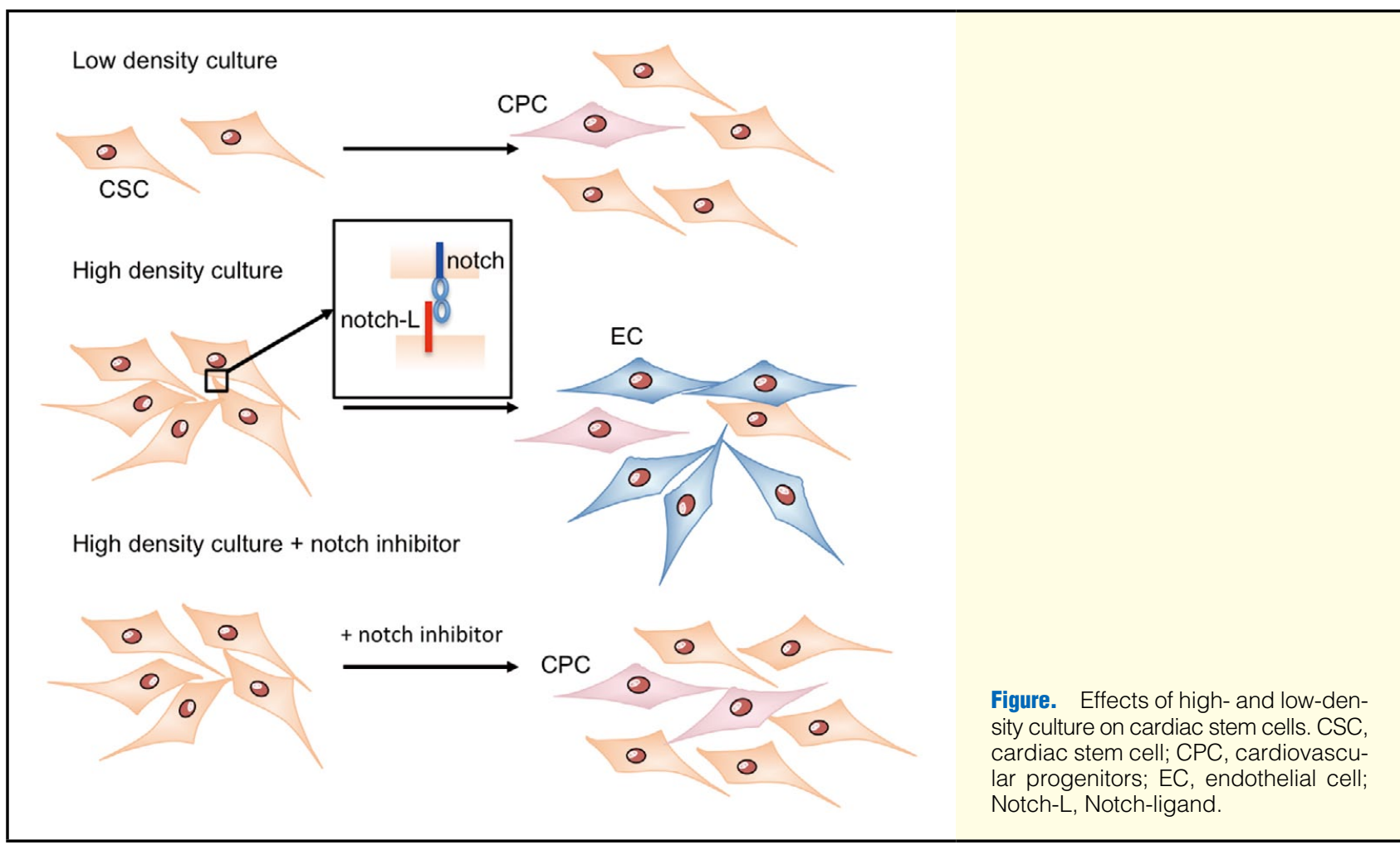

The opinions expressed in this article are not necessarily those of the editors or of the Japanese Circulation Society.

Received November 10, 2013; accepted November 10, 2013; released online November 21, 2013

Department of Signal Transduction, Research Institute for Microbial Diseases, Osaka University, Osaka; Japan Science and Technology Agency, Tokyo, Japan

Mailing address: Nobuyuki Takakura, MD, PhD, Department of Signal Transduction, Research Institute for Microbial Diseases, Osaka

University, 3-1 Yamada-oka, Suita 565-0871, Japan. E-mail: ntakaku@biken.osaka-u.ac.jp

ISSN-1346-9843 doi:10.1253/circj.CJ-13-1384

All rights are reserved to the Japanese Circulation Society. For permissions, please e-mail: cj@j-circ.or.jp 
stem cell populations are indispensable for organ regeneration.

In the field of heart failure, stem cell therapy for cardiac regeneration is creating interest. ${ }^{1}$ Initially, the existence of cardiac stem cells (CSCs) was reported in mice. ${ }^{2}$ These CSCs express c-Kit, but not hematopoietic lineage markers (Lin), and can differentiate into smooth muscle cells and endothelial cells (ECs) as well as cardiac myocytes. In humans, CSCs with a similar phenotype $\left(\mathrm{c}-\mathrm{Kit}^{+} \mathrm{Lin}^{-}\right)$have been identified in the heart. ${ }^{3} \mathrm{Ad}-$ ditionally, islet-1-positive cells have been reported to be able to differentiate into myocytes, smooth muscle cells and ECs. ${ }^{4}$ CSCs are certainly a useful source for cardiac regeneration therapy, but suffer from the drawback that they are only a very minor population in the heart. Therefore, in vitro expansion from a small piece of biopsy tissue of the heart is required. However, as with the experience with attempts to culture HSCs, expansion of stem cells without causing their differentiation has been extremely difficult.

In this issue of the Journal, Matsuda et al report that the fate of CSCs is greatly affected by cell density in culture, and by Notch signaling. ${ }^{5}$ They cultured CSCs from patients with dilated cardiomyopathy and showed that high-density cultures could not support the undifferentiated status of CSCs, which tended to differentiate into ECs under these conditions. In contrast, at low cell densities, the undifferentiated status of CSCs was maintained and their expansion was achieved. In a rat acute myocardial infarction model, injection of cells from low- but not high-density CSC cultures effectively induced regeneration of the injured heart.

What is the difference between low- and high-density culture conditions? The authors focused on the Notch signaling pathway because previous reports had suggested that this negatively affects maintenance of the undifferentiated state of CSCs. ${ }^{6}$ In low-density cultures, expression of Notch-related genes such as HES1 is suppressed; reciprocally, inhibitors of Notch signaling suppress HES1 expression in high-density cultures, resulting in maintenance of the immature status of CSCs, as in low-density cultures. The Notch ligand is a membrane-anchored protein and therefore cell-to-cell contact is important for signaling via this pathway. Under certain conditions, Notch signaling via cell-to-cell contact can be important for maintenance of the immature status of stem cell populations; however, in CSCs, Notch signaling appears to promote their differentiation, especially into ECs (Figure).

As described in this issue of the Journal, establishment of stem cell expansion methodology is important for tissue regeneration therapy. In general, very large numbers of stem cells are required for this type of therapy. To generate these large numbers of stem cells, long-term culture is required, and therefore maintenance of the stemness of the stem cells is a key consideration. To achieve this, we need to clarify how stemness of stem cells is maintained in the specific microenvironment, the so-called "niche". For example, there are 2 types of niche for HSCs in BM with opposing functions: osteoblasts support dor- mancy of HSCs, but ECs induce their self-renewal and/or differentiation. ${ }^{7}$ Interestingly, when AKT signaling is constitutively activated in ECs, they then support the stemness of HSCs. On the other hand, when the MAPK pathway is activated in ECs, they induce differentiation of HSCs. ${ }^{8}$ My group also reported similar findings that osteoblastic OP9 cells can support either stemness and expansion of HSCs or induce differentiation of HSCs when activated by EGF or bFGF, respectively. ${ }^{9}$ Taken together, it can be concluded that self-renewal and differentiation of stem cells is controlled by alternative signal pathways affecting niche cells. This knowledge can be exploited for improving methods for the in vitro expansion of stem cells. In the heart, it has been reported that CSCs localize by forming colonies together with early-differentiated immature myocytes in a pocket surrounded by mature myocytes and fibroblasts. ${ }^{10}$ These foci seem to represent a niche for CSCs; however, which cells are indeed the niche cells and which molecules are involved in maintaining CSC stemness requires further clarification. Precise molecular analysis of the CSC niche may shed light on how to improve in vitro expansion of CSCs for use in cardiac regeneration therapy.

\section{Disclosures}

None declared.

\section{References}

1. Choi SH, Jung SY, Kwon SM, Baek SH. Perspectives on stem cell therapy for cardiac regeneration. Advances and challenges. Circ J 2012; 76: 1307-1312.

2. Beltrami AP, Barlucchi L, Torella D, Baker M, Limana F, Chimenti $\mathrm{S}$, et al. Adult cardiac stem cells are multipotent and support myocardial regeneration. Cell 2003; 114: 763-776.

3. Urbanek K, Torella D, Sheikh F, De Angelis A, Nurzynska D, Silvestri $\mathrm{F}$, et al. Myocardial regeneration by activation of multipotent cardiac stem cells in ischemic heart failure. Proc Natl Acad Sci USA 2005; 102: $8692-8697$.

4. Bu L, Jiang X, Martin-Puig S, Caron L, Zhu S, Shao Y, et al. Human ISL1 heart progenitors generate diverse multipotent cardiovascular cell lineages. Nature 2009; 460: $113-117$.

5. Matsuda T, Miyagawa S, Fukushima S, Kitagawa-Sakakida S, Akimaru $\mathrm{H}$, Horii-Komatsu M, et al. Human cardiac stem cells with reduced notch signaling show enhanced therapeutic potential in a rat acute infarction model. Circ J 2014; 78: 222-231.

6. Kwon C, Qian L, Cheng P, Nigam V, Arnold J, Srivastava D. A regulatory pathway involving Notch1/beta-catenin/Isl1 determines cardiac progenitor cell fate. Nat Cell Biol 2009; 11: 951-957.

7. Ema H, Suda T. Two anatomically distinct niches regulate stem cell activity. Blood 2012; 120: 2174-2181.

8. Kobayashi H, Butler JM, O'Donnell R, Kobayashi M, Ding BS, Bonner B, et al. Angiocrine factors from Akt-activated endothelial cells balance self-renewal and differentiation of haematopoietic stem cells. Nat Cell Biol 2010; 12: 1046-1056.

9. Takakura N, Kodama H, Nishikawa S, Nishikawa S. Preferential proliferation of murine colony-forming units in culture in a chemically defined condition with a macrophage colony-stimulating factornegative stromal cell clone. J Exp Med 1996; 184: 2301-2309.

10. Urbanek K, Cesselli D, Rota M, Nascimbene A, De Angelis A, Hosoda T, et al. Stem cell niches in the adult mouse heart. Proc Natl Acad Sci USA 2006; 103: 9226-9231. 